\title{
Multiplicity for fractional differential equations with $p$-Laplacian
}

\author{
Yuansheng Tian' ${ }^{1}$, Yongfang Wei ${ }^{2}$ and Sujing Sun ${ }^{2 *}$ (D)
}

"Correspondence: kdssj@163.com

${ }^{2}$ College of Mathematics and

System Science, Shandong

University of Science and

Technology, Qingdao, P.R. China

Full list of author information is

available at the end of the article

\section{Springer}

\begin{abstract}
This paper investigates the existence of positive solution for a boundary value problem of fractional differential equations with $p$-Laplacian operator. Our analysis relies on the research of properties of the corresponding Green's function. By the use of Krasnosel'skii's fixed-point theorem, the multiplicity results of some positive solutions are obtained.
\end{abstract}

MSC: 34A08; 34B18; 35J05

Keywords: Fractional differential equation; Boundary value problem; $p$-Laplacian operator; Positive solution

\section{Introduction}

In this paper, we consider positive solutions for the following problem:

$$
\begin{aligned}
& D_{0+}^{\beta}\left(\varphi_{p}\left(D_{0+}^{\alpha} y(x)\right)\right)=f(x, y(x)), \quad 0<x<1, \\
& y(0)=y^{\prime}(0)=y(1)=D_{0+}^{\alpha} y(0)=0, \quad D_{0+}^{\alpha} y(1)=\lambda D_{0+}^{\alpha} y(\xi),
\end{aligned}
$$

where $\alpha, \beta \in \mathbb{R}, 2<\alpha \leq 3,1<\beta \leq 2$, and $\xi \in(0,1), \lambda \in[0,+\infty), \varphi_{p}(z)=|z|^{p-2} z, p>1, D_{0+}^{\alpha}$ is the Riemann-Liouville fractional derivative, and $f \in C([0,1] \times[0,+\infty),[0,+\infty))$. By using Krasnosel'skii's fixed-point theorem, we give some multiplicity results.

Differential equations of fractional order, or fractional differential equations, in which an unknown function is contained under the operation of a derivative of fractional order, have been of great interest recently. Fractional differential equation models are proved to be more adequate than integer order models for some problems in science and engineering. Many papers and books on fractional calculus and fractional differential equations have appeared recently. For an introduction of fractional calculus and fractional differential equations, we refer the reader to $[17,25]$ and the references therein. And there have been many results on existence and uniqueness of the solution of boundary value problems for fractional differential equations. For example, fractional boundary value problems at resonance $[1,5,27,39,40]$, Caputo fractional derivative problems [11, 23, 37], impulsive problems $[2,15,29,41]$, multi-point problems $[1,5,21,22,27-29,31,40]$, integral boundary value problems $[6,12,13,15]$, fractional $p$-Laplace problems $[8,10,14,21,22,35,36]$, fractional lower and upper solution problems $[4,7,30,38]$, fractional delay problems, [24, 33, 34], solitons [9], singular problems [3], etc.

(c) The Author(s) 2018. This article is distributed under the terms of the Creative Commons Attribution 4.0 International License (http://creativecommons.org/licenses/by/4.0/), which permits unrestricted use, distribution, and reproduction in any medium, provided you give appropriate credit to the original author(s) and the source, provide a link to the Creative Commons license, and indicate if changes were made. 
On the other hand, integer order differential equations with $p$-Laplacian operator also arise in different research areas such as physical and natural phenomena, non-Newtonian mechanics, nonlinear elasticity and glaciology, combustion theory, population biology, nonlinear flow laws, and system of Monge-Kantorovich partial differential equations [8, $16,19,20,32]$. For example, turbulent flow in a porous medium is a fundamental mechanics problem. For studying this type of problem, Leibenson [19] introduced differential equations with $p$-Laplacian operator

$$
\left(\varphi_{p}\left(y^{\prime}(x)\right)\right)^{\prime}+f(x, y(x))=0
$$

In [26], by applying the fixed point index theory, Su studied the existence of positive solutions of a nonlinear four-point singular boundary value problem with a $p$-Laplacian operator:

$$
\begin{cases}\left(\varphi_{p}\left(y^{\prime}(x)\right)\right)^{\prime}+a(x) f(y(x))=0, & 0<x<1 \\ \alpha \varphi_{p}(y(0))-\beta \varphi_{p}\left(y^{\prime}(\xi)\right)=0, & \gamma \varphi_{p}(y(1))-\delta \varphi_{p}\left(y^{\prime}(\eta)\right)=0\end{cases}
$$

It is quite natural to study fractional differential equation relative to equation. Recently, many scholars have paid more attention to the fractional order differential equation boundary value problems with $p$-Laplacian operator, see [8, 10, 14, 21, 22]. Recently, Dong et al. [10] investigated the following $p$-Laplacian fractional differential equation boundary value problem:

$$
\begin{aligned}
& D^{\alpha}\left(\varphi_{p}\left(D^{\alpha} y(x)\right)\right)=f(x, y(x)), \quad 0<x<1, \\
& y(0)=y(1)=D^{\alpha} y(0)=D^{\alpha} y(1)=0,
\end{aligned}
$$

where $1<\alpha \leq 2$ is a real number, $D^{\alpha}$ is the conformable fractional derivative. Some existence and multiplicity results of positive solutions are proved by the fixed-point theorems on cone. The purpose of this paper is to generalize some existence results of the above references to a nonlinear fractional boundary value problem with $p$-Laplacian.

\section{Preliminaries}

Definition 2.1 ([17]) The fractional integral of order $\alpha>0$ of a function $y:(0,+\infty) \rightarrow \mathbb{R}$ is defined as

$$
I_{0+}^{\alpha} y(x)=\frac{1}{\Gamma(\alpha)} \int_{0}^{x}(x-z)^{\alpha-1} y(z) d z
$$

Definition 2.2 ([17]) The fractional derivative of order $\alpha>0$ of a continuous function $y:(0,+\infty) \rightarrow \mathbb{R}$ is defined as

$$
D_{0+}^{\alpha} y(x)=\frac{1}{\Gamma(n-\alpha)}\left(\frac{d}{d x}\right)^{n} \int_{0}^{x} \frac{y(z)}{(x-z)^{\alpha-n+1}} d z,
$$

where $n=-[-\alpha]$. 
Lemma 2.1 ([17]) Assume that $y, D_{0+}^{\alpha} y \in C(0,1) \cap L(0,1)$. Then

$$
I_{0+}^{\alpha} D_{0+}^{\alpha} y(x)=y(x)+c_{1} x^{\alpha-1}+c_{2} x^{\alpha-2}+\cdots+c_{N} x^{\alpha-N}, \quad c_{i} \in \mathbb{R}, i=1,2, \ldots, N
$$

where $N=-[-\alpha]$.

Let $\mathcal{M}:=\lambda^{p-1} \xi^{\beta-1}$ such that $\mathcal{M} \neq 1$, and

$$
\begin{aligned}
& G(x, z)= \begin{cases}\frac{1}{\Gamma(\alpha)}[x(1-z)]^{\alpha-1}, & 0 \leq x \leq z \leq 1 ; \\
\frac{1}{\Gamma(\alpha)}\left([x(1-z)]^{\alpha-1}-(x-z)^{\alpha-1}\right), & 0 \leq z \leq x \leq 1,\end{cases} \\
& H(x, z)= \begin{cases}\frac{[x(1-z)]^{\beta-1}-\lambda^{p-1}[x(\xi-z)]^{\beta-1}-(1-\mathcal{M})(x-z)^{\beta-1}}{(1-\mathcal{M}) \Gamma(\beta)}, & 0 \leq z \leq x \leq 1, z \leq \xi ; \\
\frac{[x(1-z)]^{\beta-1}-(1-\mathcal{M})(x-z)^{\beta-1}}{(1-\mathcal{M}) \Gamma(\beta)}, & 0<\xi \leq z \leq x \leq 1 ; \\
\frac{[x(1-z)]^{\beta-1}-\lambda^{p-1}[x(\xi-z)]^{\beta-1}}{(1-\mathcal{M}) \Gamma(\beta)}, & 0 \leq x \leq z \leq \xi<1 ; \\
\frac{[x(1-z)]^{\beta-1}}{(1-\mathcal{M}) \Gamma(\beta)}, & 0 \leq x \leq z \leq 1, \xi \leq z .\end{cases}
\end{aligned}
$$

Lemma 2.2 Let $Q(x)=x(1-x)^{\alpha-1}$. Then functions $G(x, z), H(x, z) \in C([0,1] \times[0,1])$ and satisfy:

(1) $G(x, z)=G(1-z, 1-x) ; G(x, z)>0$ for $x, z \in(0,1)$;

(2) $\frac{Q(1-x) Q(z)}{\Gamma(\alpha)} \leq G(x, z) \leq \frac{(\alpha-1) Q(z)}{\Gamma(\alpha)}$ for $x, z \in[0,1]$;

(3) If $\mathcal{M}<1$, then $H(x, z)>0$ for $x, z \in(0,1)$.

Proof It is easily seen that functions $G(x, z), H(x, z) \in C([0,1] \times[0,1])$ and $(1)$ hold. We will only prove (2) and (3).

(2) For $0 \leq z \leq x \leq 1$, since $0<\alpha-2 \leq 1$, one has

$$
\begin{aligned}
G(x, z) & =\frac{1}{\Gamma(\alpha)}\left([x(1-z)]^{\alpha-1}-(x-z)^{\alpha-1}\right) \\
& =\frac{\alpha-1}{\Gamma(\alpha)} \int_{x-z}^{x(1-z)} s^{\alpha-2} d s \\
& \leq \frac{\alpha-1}{\Gamma(\alpha)}[x(1-z)]^{\alpha-2}[x(1-z)-(x-z)] \\
& \leq \frac{\alpha-1}{\Gamma(\alpha)}(1-z)^{\alpha-2} z(1-x) \\
& \leq \frac{(\alpha-1) Q(z)}{\Gamma(\alpha)},
\end{aligned}
$$

and

$$
\begin{aligned}
G(x, z) & =\frac{1}{\Gamma(\alpha)}\left([x(1-z)]^{\alpha-1}-(x-z)^{\alpha-1}\right) \\
& \left.\geq \frac{1}{\Gamma(\alpha)}\left([x(1-z)]^{\alpha-2}[x(1-z)]-(x-z)\right]\right) \\
& =\frac{1}{\Gamma(\alpha)}[x(1-z)]^{\alpha-2} z(1-x) \\
& \geq \frac{1}{\Gamma(\alpha)} x^{\alpha-1}(1-z)^{\alpha-1} z(1-x)
\end{aligned}
$$




$$
=\frac{Q(z) Q(1-x)}{\Gamma(\alpha)} .
$$

For $0 \leq x \leq z \leq 1$, one has

$$
\begin{aligned}
G(x, z) & =\frac{1}{\Gamma(\alpha)} x^{\alpha-1}(1-z)^{\alpha-1} \\
& \leq \frac{1}{\Gamma(\alpha)} z^{\alpha-1}(1-z)^{\alpha-1} \\
& \leq \frac{1}{\Gamma(\alpha)}(\alpha-1) z(1-z)^{\alpha-1} \\
& \leq \frac{(\alpha-1) Q(z)}{\Gamma(\alpha)}
\end{aligned}
$$

and

$$
\begin{aligned}
G(x, z) & =\frac{1}{\Gamma(\alpha)} x^{\alpha-1}(1-z)^{\alpha-1} \\
& \geq \frac{1}{\Gamma(\alpha)} x^{\alpha-1}(1-z)^{\alpha-1} z(1-x) \\
& =\frac{Q(z) Q(1-x)}{\Gamma(\alpha)} .
\end{aligned}
$$

So, $\frac{Q(1-z) Q(z)}{\Gamma(\alpha)} \leq G(x, z) \leq \frac{(\alpha-1) Q(z)}{\Gamma(\alpha)}$ for $x, z \in[0,1]$.

(3) For $0<z \leq x<1, z \leq \xi$, set

$$
h(x, z)=\frac{[x(1-z)]^{\beta-1}-(x-z)^{\beta-1}}{\Gamma(\beta)} .
$$

It is obvious that $h(x, z)>0$ for $0<z \leq x<1$. Hence, we get

$$
\begin{aligned}
H(x, z) & =\frac{[x(1-z)]^{\beta-1}-\lambda^{p-1}[x(\xi-z)]^{\beta-1}-(1-\mathcal{M})(x-z)^{\beta-1}}{(1-\mathcal{M}) \Gamma(\beta)} \\
& =\left(1+\frac{\mathcal{M}}{1-\mathcal{M}}\right) \frac{[x(1-z)]^{\beta-1}}{\Gamma(\beta)}-\frac{(x-z)^{\beta-1}}{\Gamma(\beta)}-\frac{\lambda^{p-1}[x(\xi-z)]^{\beta-1}}{(1-\mathcal{M}) \Gamma(\beta)} \\
& =\frac{[x(1-z)]^{\beta-1}-(x-z)^{\beta-1}}{\Gamma(\beta)}+\frac{\lambda^{p-1} x^{\beta-1}\left[\xi^{\beta-1}(1-z)^{\beta-1}-(\xi-z)^{\beta-1}\right]}{(1-\mathcal{M}) \Gamma(\beta)} \\
& =h(x, z)+\frac{\lambda^{p-1} x^{\beta-1}}{1-\mathcal{M}} h(\xi, z) \\
& >0 .
\end{aligned}
$$

Similarly, there holds $H(x, z)>0$ for $0<\eta \leq z \leq x<1$ or $0<x \leq z \leq \eta<1$ or $0<x \leq z<$ $1, \eta \leq z$.

Thus, $H(x, z)>0$ for $x, z \in(0,1)$. The proof is completed. 
Lemma 2.3 Suppose that $h \in C[0,1], p>1, \alpha, \beta \in \mathbb{R}, 2<\alpha \leq 3,1<\beta \leq 2$ and $\xi \in(0,1), \lambda \in$ $[0,+\infty)$. Then the following problem

$$
\left\{\begin{array}{l}
D_{0+}^{\beta}\left(\varphi_{p}\left(D_{0+}^{\alpha} y(x)\right)\right)=y(x), \quad 0<x<1, \\
y(0)=y^{\prime}(0)=y(1)=D_{0+}^{\alpha} y(0)=0, \quad D_{0+}^{\alpha} y(1)=\lambda D_{0+}^{\alpha} y(\xi),
\end{array}\right.
$$

has a unique solution

$$
y(x)=\int_{0}^{1} G(x, z) \varphi_{q}\left(\int_{0}^{1} H(z, \tau) y(\tau) d \tau\right) d z,
$$

where $\varphi_{q}=\left(\varphi_{p}\right)^{-1}, \frac{1}{p}+\frac{1}{q}=1$.

Proof From the equation of (2.2), Lemma 2.1, and the fact that $D_{0+}^{\alpha} y(0)=0$, there is

$$
\varphi_{p}\left(D_{0+}^{\alpha} y(x)\right)=\frac{1}{\Gamma(\beta)} \int_{0}^{x}(x-z)^{\beta-1} y(z) d z+c_{1} x^{\beta-1}
$$

for some $c_{1} \in \mathbb{R}$. Thus,

$$
\begin{aligned}
& \varphi_{p}\left(D_{0+}^{\alpha} y(1)\right)=\frac{1}{\Gamma(\beta)} \int_{0}^{1}(1-z)^{\beta-1} y(z) d z+c_{1}, \\
& \varphi_{p}\left(D_{0_{+}}^{\alpha} y(\xi)\right)=\frac{1}{\Gamma(\beta)} \int_{0}^{\xi}(\xi-z)^{\beta-1} y(z) d z+c_{1} \xi^{\beta-1} .
\end{aligned}
$$

Taking into account that $D_{0+}^{\alpha} y(1)=\lambda D_{0+}^{\alpha} y(\xi)$, combining with (2.5) and (2.6), we obtain

$$
c_{1}=-\int_{0}^{1} \frac{(1-z)^{\beta-1}}{\Gamma(\beta)(1-\mathcal{M})} y(z) d z+\int_{0}^{\xi} \frac{\lambda^{p-1}(\xi-z)^{\beta-1}}{\Gamma(\beta)(1-\mathcal{M})} y(z) d z
$$

Thus,

$$
\begin{aligned}
\varphi_{p}\left(D_{0+}^{\alpha} y(x)\right)= & \int_{0}^{x} \frac{(x-z)^{\beta-1}}{\Gamma(\beta)} y(z) d z-\int_{0}^{1} \frac{x^{\beta-1}(1-z)^{\beta-1}}{\Gamma(\beta)(1-\mathcal{M})} y(z) d z \\
& +\int_{0}^{\xi} \frac{\lambda^{p-1} x^{\beta-1}(\xi-z)^{\beta-1}}{\Gamma(\beta)(1-\mathcal{M})} y(z) d z \\
= & -\int_{0}^{1} H(x, z) y(z) d z
\end{aligned}
$$

then

$$
D_{0+}^{\alpha} y(x)+\varphi_{q}\left(\int_{0}^{1} H(x, z) y(z) d z\right)=0 .
$$

By the use of Lemma 2.1, Eq. (2.7) is equivalent to the integral equation

$$
y(x)=-I_{0+}^{\alpha} \varphi_{q}\left(\int_{0}^{1} H(x, z) y(z) d z\right)+d_{1} x^{\alpha-1}+d_{2} x^{\alpha-2}+d_{3} x^{\alpha-3}
$$

for some $d_{1}, d_{2}, d_{3} \in \mathbb{R}$. 
By $y(0)=y^{\prime}(0)=0$, there are $d_{2}=d_{3}=0$. Thus

$$
\begin{aligned}
y(x) & =-I_{0+}^{\alpha} \varphi_{q}\left(\int_{0}^{1} H(x, z) y(z) d z\right)+d_{1} x^{\alpha-1} \\
& =-\frac{1}{\Gamma(\alpha)} \int_{0}^{x}(x-z)^{\alpha-1} \varphi_{q}\left(\int_{0}^{1} H(z, \tau) y(\tau) d \tau\right) d z+d_{1} x^{\alpha-1} .
\end{aligned}
$$

By $y(1)=0$, there is

$$
d_{1}=\frac{1}{\Gamma(\alpha)} \int_{0}^{1}(1-z)^{\alpha-1} \varphi_{q}\left(\int_{0}^{1} H(z, \tau) y(\tau) d \tau\right) d z
$$

Therefore, the unique solution of problem (2.2) is

$$
\begin{aligned}
y(x)= & -\frac{1}{\Gamma(\alpha)} \int_{0}^{x}(x-z)^{\alpha-1} \varphi_{q}\left(\int_{0}^{1} H(z, \tau) y(\tau) d \tau\right) d z \\
& +\frac{1}{\Gamma(\alpha)} \int_{0}^{1}[x(1-z)]^{\alpha-1} \varphi_{q}\left(\int_{0}^{1} H(z, \tau) y(\tau) d \tau\right) d z \\
= & \int_{0}^{1} G(x, z) \varphi_{q}\left(\int_{0}^{1} H(z, \tau) y(\tau) d \tau\right) d z .
\end{aligned}
$$

The proof is completed.

Let $E=C[0,1]$ be a Banach space with the maximum norm $\|y\|=\max _{0 \leq x \leq 1}|y(x)|$. Define a cone $P \subset E$ by

$$
P=\left\{y \in E \mid y(x) \geq \frac{Q(1-x)}{\alpha-1}\|y\|, 0 \leq x \leq 1\right\} .
$$

Lemma 2.4 Define $\mathcal{T}: P \rightarrow$ E as

$$
(\mathcal{T} y)(x)=\int_{0}^{1} G(x, z) \varphi_{q}\left(\int_{0}^{1} H(z, \tau) f(\tau, y(\tau)) d \tau\right) d z
$$

Then $\mathcal{T}: P \rightarrow P$ is a completely continuous operator.

Proof By the use of relation (2) of Lemma 2.2, for any $y \in P$, there hold

$$
(\mathcal{T} y)(x) \leq \frac{\alpha-1}{\Gamma(\alpha)} \int_{0}^{1} Q(z) \varphi_{q}\left(\int_{0}^{1} H(z, \tau) f(\tau, y(\tau)) d \tau\right) d z
$$

and

$$
(\mathcal{T} y)(x) \geq \frac{Q(1-x)}{\Gamma(\alpha)} \int_{0}^{1} Q(z) \varphi_{q}\left(\int_{0}^{1} H(z, \tau) f(\tau, y(\tau)) d \tau\right) d z
$$

Then $(\mathcal{T} y)(x) \geq \frac{q(1-x)}{\alpha-1}\|\mathcal{T} y\|$, which implies $\mathcal{T}: P \rightarrow P$. By the use of the Arzela-Ascoli theorem, a standard proof shows that $\mathcal{T}: P \rightarrow P$ is completely continuous. 
Lemma 2.5 ([18]) Let $E$ be an ordered Banach space, $P \subset E$ be a cone. Suppose that $\Omega_{1}$, $\Omega_{2}$ are bounded open subsets of $E$ with $0 \in \Omega_{1} \subset \bar{\Omega}_{1} \subset \Omega_{2}$, and $\mathcal{T}: P \rightarrow P$ is a completely continuous operator such that either

$\left(A_{1}\right)\|\mathcal{T} y\| \leq\|y\|, y \in P \cap \partial \Omega_{1}$ and $\|\mathcal{T} y\| \geq\|y\|, y \in P \cap \partial \Omega_{2}$, or

$\left(A_{2}\right)\|\mathcal{T} y\| \geq\|y\|, y \in P \cap \partial \Omega_{1}$ and $\|\mathcal{T} y\| \leq\|y\|, y \in P \cap \partial \Omega_{2}$.

Then $\mathcal{T}$ has a fixed point in $P \cap \bar{\Omega}_{2} \backslash \Omega_{1}$.

\section{Existence of positive solutions}

For notational convenience, denote

$$
\begin{aligned}
f_{0} & =\liminf _{y \rightarrow+0} \min _{x \in[1 / 4,3 / 4]} \frac{f(x, y)}{y^{p-1}}, \quad f^{0}=\limsup _{y \rightarrow+0} \max _{x \in[0,1]} \frac{f(x, y)}{y^{p-1}}, \\
f_{\infty} & =\liminf _{y \rightarrow+\infty} \min _{x \in[1 / 4,3 / 4]} \frac{f(x, y)}{y^{p-1}}, \quad f^{\infty}=\limsup _{y \rightarrow+\infty} \max _{x \in[0,1]} \frac{f(x, y)}{y^{p-1}}, \\
\rho_{*} & =\left(\frac{\alpha-1}{\Gamma(\alpha)} \int_{0}^{1} Q(z) \varphi_{q}\left(\int_{0}^{1} H(z, \tau) d \tau\right) d z\right)^{-1}, \\
\rho^{*} & =\left(\frac{Q\left(\frac{1}{2}\right)}{\Gamma(\alpha)} \int_{0}^{1} Q(z) \varphi_{q}\left(\int_{\frac{1}{4}}^{\frac{3}{4}} H(z, \tau) d \tau\right) d z\right)^{-1}, \quad \sigma=\min _{1 / 4 \leq x \leq 3 / 4} \frac{Q(1-x)}{\alpha-1} .
\end{aligned}
$$

From now on we will use the following assumptions:

$\left(C_{1}\right) f_{0} \in\left(\left(\frac{\rho^{*}}{\sigma}\right)^{p-1}, \infty\right], f_{\infty} \in\left(\left(\frac{\rho^{*}}{\sigma}\right)^{p-1}, \infty\right]$.

$\left(C_{2}\right) f^{0} \in\left[0, \rho_{*}^{p-1}\right), f^{\infty} \in\left[0, \rho_{*}^{p-1}\right)$.

$\left(C_{3}\right)$ There exist constants $d \in\left(0, \rho_{*}\right)$ and $\lambda_{1}>0$ such that

$$
f(x, y) \leq\left(d \lambda_{1}\right)^{p-1}, \quad 0 \leq x \leq 1,0 \leq y \leq \lambda_{1}
$$

$\left(C_{4}\right)$ There exist constants $D \in\left(\rho^{*}, \infty\right)$ and $\lambda_{2}>0$ such that

$$
f(x, y) \geq\left(D \lambda_{2}\right)^{p-1}, \quad 1 / 4 \leq x \leq 3 / 4, \sigma \lambda_{2} \leq y \leq \lambda_{2}
$$

Theorem 3.1 Assume that conditions $\left(C_{1}\right),\left(C_{3}\right)$ hold, then problem (1.1) has at least two solutions $y_{1}$ and $y_{2}$ such that $0<\left\|y_{1}\right\|<\lambda_{1}<\left\|y_{2}\right\|$.

Proof Firstly, by condition $\left(C_{3}\right)$, there exist constants $d \in\left(0, \rho_{*}\right)$ and $\lambda_{1}>0$ such that

$$
f(x, y) \leq\left(d \lambda_{1}\right)^{p-1}, \quad 0 \leq x \leq 1,0 \leq y \leq \lambda_{1} .
$$

Set $\Omega_{\lambda_{1}}=\left\{y \in P \mid\|y\|<\lambda_{1}\right\}$. Taking into account the monotonicity of $\varphi(z)$ and relation (2) of Lemma 2.2, for $y \in \partial \Omega_{\lambda_{1}}$, we have

$$
\begin{aligned}
\|\mathcal{T} y\| & =\max _{0 \leq x \leq 1}|\mathcal{T} y(x)| \\
& \leq \frac{\alpha-1}{\Gamma(\alpha)} \int_{0}^{1} Q(z) \varphi_{q}\left(\int_{0}^{1} H(z, \tau) f(\tau, y(\tau)) d \tau\right) d z \\
& \leq \frac{\alpha-1}{\Gamma(\alpha)} \int_{0}^{1} Q(z) \varphi_{q}\left(\int_{0}^{1} H(z, \tau)\left(d \lambda_{1}\right)^{(p-1)} d \tau\right) d z
\end{aligned}
$$




$$
\begin{aligned}
& \leq \rho_{*} \lambda_{1} \frac{\alpha-1}{\Gamma(\alpha)} \int_{0}^{1} Q(z) \varphi_{q}\left(\int_{0}^{1} H(z, \tau) d \tau\right) d z \\
& =\lambda_{1}=\|y\| .
\end{aligned}
$$

Thus, $\|\mathcal{T} y\| \leq\|y\|$ for all $y \in \partial \Omega_{\lambda_{1}}$.

Secondly, with the first relation of condition $\left(C_{1}\right), f_{0} \in\left(\left(\frac{\rho^{*}}{\sigma}\right)^{p-1}, \infty\right)$, there exists a real number $r_{1} \in\left(0, \lambda_{1}\right)$ such that

$$
f(x, y) \geq y^{p-1}\left(\frac{\rho^{*}}{\sigma}\right)^{p-1}, \quad \text { for } \frac{1}{4} \leq x \leq \frac{3}{4}, 0<y \leq r_{1} .
$$

Set $\Omega_{r_{1}}=\left\{y \in P \mid\|y\|<r_{1}\right\}$. For $y \in \partial \Omega_{r_{1}}$, we have

$$
r_{1}=\|y\| \geq y(x) \geq \frac{Q(1-x)}{\alpha-1}\|y\| \geq \sigma\|y\|=\sigma r_{1}, \quad x \in\left[\frac{1}{4}, \frac{3}{4}\right] .
$$

Thus, with relation (2) of Lemma 2.2, there is

$$
\begin{aligned}
\|\mathcal{T} y\| & =\max _{0 \leq x \leq 1}|\mathcal{T} y(x)| \\
& =\max _{0 \leq x \leq 1} \int_{0}^{1} G(x, z) \varphi_{q}\left(\int_{0}^{1} H(z, \tau) f(\tau, y(\tau)) d \tau\right) d z \\
& \geq \int_{0}^{1} G\left(\frac{1}{2}, z\right) \varphi_{q}\left(\int_{\frac{1}{4}}^{\frac{3}{4}} H(z, \tau) y^{p-1}(\tau)\left(\frac{\rho^{*}}{\sigma}\right)^{p-1} d \tau\right) d z \\
& \geq \frac{r_{1} \rho^{*}}{\Gamma(\alpha)} \int_{0}^{1} Q\left(\frac{1}{2}\right) Q(z) \varphi_{q}\left(\int_{\frac{1}{4}}^{\frac{3}{4}} H(z, \tau) d \tau\right) d z \\
& =r_{1}=\|y\| .
\end{aligned}
$$

So, $\|\mathcal{T} y\| \geq\|y\|$ for all $y \in \partial \Omega_{r_{1}}$.

Thirdly, with the second relation of condition $\left(C_{1}\right), f_{\infty} \in\left(\left(\frac{\rho^{*}}{\sigma}\right)^{p-1}, \infty\right)$, there exists a real number $R^{*}>0$ such that

$$
f(x, y) \geq y^{p-1}\left(\frac{\rho^{*}}{\sigma}\right)^{p-1}, \quad \text { for } \frac{1}{4} \leq x \leq \frac{3}{4}, y \geq R^{*} .
$$

Choose $R_{1}=\max \left\{2 \lambda_{1}, \frac{R^{*}}{\sigma}\right\}$, set $\Omega_{R_{1}}=\left\{y \in P \mid\|y\|<R_{1}\right\}$. For $y \in \partial \Omega_{R_{1}}$, we get

$$
R_{1}=\|y\| \geq y(x) \geq \frac{Q(1-x)}{\alpha-1}\|y\| \geq \sigma\|y\|=\sigma R_{1} \geq R^{*}, \quad x \in\left[\frac{1}{4}, \frac{3}{4}\right] .
$$

Thus, with relation (2) of Lemma 2.2, there is

$$
\begin{aligned}
\|\mathcal{T} y\| & =\max _{0 \leq x \leq 1}|\mathcal{T} y(x)| \\
& =\max _{0 \leq x \leq 1} \int_{0}^{1} G(x, z) \varphi_{q}\left(\int_{0}^{1} H(z, \tau) f(\tau, y(\tau)) d \tau\right) d z \\
& \geq \int_{0}^{1} G\left(\frac{1}{2}, z\right) \varphi_{q}\left(\int_{\frac{1}{4}}^{\frac{3}{4}} H(z, \tau) u^{p-1}(\tau)\left(\frac{\rho^{*}}{\sigma}\right)^{p-1} d \tau\right) d z
\end{aligned}
$$




$$
\begin{aligned}
& \geq \frac{R_{1} \rho^{*}}{\Gamma(\alpha)} \int_{0}^{1} Q\left(\frac{1}{2}\right) Q(z) \varphi_{q}\left(\int_{\frac{1}{4}}^{\frac{3}{4}} H(z, \tau) d \tau\right) d z \\
& =R_{1}=\|y\| .
\end{aligned}
$$

So, $\|\mathcal{T} y\| \geq\|y\|$ for all $y \in \partial \Omega_{R_{1}}$.

By Lemma 2.5, $\mathcal{T}$ has a fixed point $y_{1} \in\left(\Omega_{\lambda_{1}} \backslash \bar{\Omega}_{r_{1}}\right)$ and a fixed point $y_{2} \in\left(\Omega_{R_{1}} \backslash \bar{\Omega}_{\lambda_{1}}\right)$. That is to say, $y_{1}, y_{2}$ are both positive solutions of problem (1.1) such that $0<\left\|y_{1}\right\|<\lambda_{1}<\left\|y_{2}\right\|$.

Theorem 3.2 Assume that conditions $\left(C_{2}\right),\left(C_{4}\right)$ hold, then problem (1.1) has at least two solutions $y_{1}$ and $y_{2}$ satisfying $0<\left\|y_{1}\right\|<\lambda_{2}<\left\|y_{2}\right\|$.

Proof Firstly, by condition $\left(C_{4}\right)$, there exist two constants $D \in\left(\rho^{*}, \infty\right)$ and $\lambda_{2}>0$ such that

$$
f(x, y) \geq\left(D \lambda_{2}\right)^{p-1}, \quad 1 / 4 \leq x \leq 3 / 4, \sigma \lambda_{2} \leq y \leq \lambda_{2}
$$

Set $\Omega_{\lambda_{2}}=\left\{y \in P \mid\|y\|<\lambda_{2}\right\}$. For $y \in \partial \Omega_{\lambda_{2}}$, one has

$$
\lambda_{2}=\|y\| \geq y(x) \geq \frac{Q(1-x)}{\alpha-1}\|y\| \geq \sigma\|y\|=\sigma \lambda_{2}, \quad x \in\left[\frac{1}{4}, \frac{3}{4}\right] .
$$

Thus, we get

$$
\begin{aligned}
\|\mathcal{T} y\| & =\max _{0 \leq x \leq 1}|T y(x)| \\
& =\max _{0 \leq x \leq 1} \int_{0}^{1} G(x, z) \varphi_{q}\left(\int_{0}^{1} H(z, \tau) f(\tau, y(\tau)) d \tau\right) d z \\
& \geq \int_{0}^{1} G\left(\frac{1}{2}, z\right) \varphi_{q}\left(\int_{\frac{1}{4}}^{\frac{3}{4}} H(z, \tau)\left(D \lambda_{2}\right)^{(p-1)} d \tau\right) d z \\
& \geq \frac{\lambda_{2} \rho^{*}}{\Gamma(\alpha)} \int_{0}^{1} Q\left(\frac{1}{2}\right) Q(z) \varphi_{q}\left(\int_{\frac{1}{4}}^{\frac{3}{4}} H(z, \tau) d \tau\right) d z \\
& =\lambda_{2}=\|y\| .
\end{aligned}
$$

So, $\|\mathcal{T} y\| \geq\|y\|$ for all $y \in \partial \Omega_{\lambda_{2}}$.

Secondly, with the first relation of condition $\left(C_{2}\right), f^{0} \in\left[0, \rho_{*}^{p-1}\right)$, there exists a real number $r_{2} \in\left(0, \lambda_{2}\right)$ such that

$$
f(x, y) \leq y^{p-1} \rho_{*}^{p-1} \leq\left(r_{2} \rho_{*}\right)^{p-1}, \quad \text { for } 0 \leq x \leq 1,0<y \leq r_{2} .
$$

Set $\Omega_{r_{2}}=\left\{y \in P \mid\|y\|<r_{2}\right\}$. For $y \in \partial \Omega_{r_{2}}$, one has

$$
\begin{aligned}
\|\mathcal{T} y\| & =\max _{0 \leq x \leq 1}|T y(x)| \\
& \leq \frac{\alpha-1}{\Gamma(\alpha)} \int_{0}^{1} Q(z) \varphi_{q}\left(\int_{0}^{1} H(z, \tau) f(\tau, y(\tau)) d \tau\right) d z \\
& \leq \frac{\alpha-1}{\Gamma(\alpha)} \int_{0}^{1} Q(z) \varphi_{q}\left(\int_{0}^{1} H(z, \tau)\left(r_{2} \rho_{*}\right)^{p-1} d \tau\right) d z
\end{aligned}
$$




$$
\begin{aligned}
& =r_{2} \rho_{*} \frac{\alpha-1}{\Gamma(\alpha)} \int_{0}^{1} Q(z) \varphi_{q}\left(\int_{0}^{1} H(z, \tau) d \tau\right) d z \\
& =r_{2}=\|y\| .
\end{aligned}
$$

So, $\|\mathcal{T} y\| \leq\|y\|$ for all $y \in \partial \Omega_{r_{2}}$.

Thirdly, with the second relation of condition $\left(C_{2}\right), f^{\infty} \in\left[0, \rho_{*}^{p-1}\right)$, there exists a positive number $R^{*}$ such that

$$
f(x, y) \leq y^{p-1} \rho_{*}^{p-1}, \quad \text { for } 0 \leq x \leq 1, y \geq R^{*} .
$$

We now consider two situations.

Case 1. The function $f$ is bounded on $[0, \infty)$. We can choose a positive number $G>0$ such that $f(x, y) \leq G^{p-1} \rho_{*}^{p-1}$ for $x \in[0,1], y \in[0, \infty)$. Let $R_{2}=\max \left\{2 \lambda_{2}, G\right\}$ and $\Omega_{R_{2}}=\{y \in$ $\left.P \mid\|y\|<R_{2}\right\}$. For $y \in \partial \Omega_{R_{2}}$, one has

$$
\begin{aligned}
\|\mathcal{T} y\| & =\max _{0 \leq x \leq 1}|\mathcal{T} y(x)| \\
& \leq \frac{\alpha-1}{\Gamma(\alpha)} \int_{0}^{1} Q(z) \varphi_{q}\left(\int_{0}^{1} H(z, \tau) f(\tau, y(\tau)) d \tau\right) d z \\
& \leq \frac{\alpha-1}{\Gamma(\alpha)} \int_{0}^{1} Q(z) \varphi_{q}\left(\int_{0}^{1} H(z, \tau) G^{p-1} \rho_{*}^{p-1} d \tau\right) d z \\
& \leq R_{2} \rho_{*} \frac{\alpha-1}{\Gamma(\alpha)} \int_{0}^{1} Q(z) \varphi_{q}\left(\int_{0}^{1} H(z, \tau) d \tau\right) d z \\
& =R_{2}=\|y\| .
\end{aligned}
$$

So, $\|\mathcal{T} y\| \leq\|y\|$ for all $y \in \partial \Omega_{R_{2}}$.

Case 2. The function $f$ is unbounded on $[0, \infty)$. We can choose a positive number $R_{2}>$ $\max \left\{2 \lambda_{2}, R^{*}\right\}$ such that $f(x, y) \leq f\left(x, R_{2}\right)$ for $x \in[0,1], y \in\left(0, R_{2}\right)$. Set $\Omega_{R_{2}}=\{y \in P \mid\|y\|<$ $\left.R_{2}\right\}$. For $y \in \partial \Omega_{R_{2}}$, one has

$$
\begin{aligned}
\|\mathcal{T} y\| & =\max _{0 \leq x \leq 1}|\mathcal{T} y(x)| \\
& \leq \frac{\alpha-1}{\Gamma(\alpha)} \int_{0}^{1} Q(z) \varphi_{q}\left(\int_{0}^{1} H(z, \tau) f(\tau, y(\tau)) d \tau\right) d z \\
& \leq \frac{\alpha-1}{\Gamma(\alpha)} \int_{0}^{1} Q(z) \varphi_{q}\left(\int_{0}^{1} H(z, \tau) f\left(\tau, R_{2}\right) d \tau\right) d z \\
& \leq \frac{\alpha-1}{\Gamma(\alpha)} \int_{0}^{1} Q(z) \varphi_{q}\left(\int_{0}^{1} H(z, \tau) R_{2}^{p-1} \rho_{*}^{p-1} d \tau\right) d z \\
& \leq R_{2} \rho_{*} \frac{\alpha-1}{\Gamma(\alpha)} \int_{0}^{1} Q(z) \varphi_{q}\left(\int_{0}^{1} H(z, \tau) d \tau\right) d z \\
& =R_{2}=\|y\| .
\end{aligned}
$$

So, $\|\mathcal{T} y\| \leq\|y\|$ for all $y \in \partial \Omega_{R_{2}}$.

By Lemma 2.5, $\mathcal{T}$ has a fixed point $y_{1} \in\left(\Omega_{\lambda_{2}} \backslash \bar{\Omega}_{r_{2}}\right)$ and a fixed point $y_{2} \in\left(\Omega_{R_{2}} \backslash \bar{\Omega}_{\lambda_{2}}\right)$. That is to say, $y_{1}, y_{2}$ are both positive solutions of problem (1.1) and $0<\left\|y_{1}\right\|<\lambda_{2}<$ $\left\|y_{2}\right\|$. 
By Theorems 3.1 and 3.2, we can obtain the following corollary.

Corollary 3.1 Problem (1.1) has at least one positive solution if one of the following assumptions is satisfied:

$(A 1)$ Conditions $\left(C_{3}\right)$ and $\left(C_{4}\right)$ hold; or

(A2) Conditions $f^{0} \in\left[0, \rho_{*}^{p-1}\right)$ and $f_{\infty} \in\left(\left(\frac{\rho^{*}}{\sigma}\right)^{p-1}, \infty\right)$ hold; or

(A3) Conditions $f_{0} \in\left(\left(\frac{\rho^{*}}{\sigma}\right)^{p-1}, \infty\right)$ and $f^{\infty} \in\left[0, \rho_{*}^{p-1}\right)$ hold; or

$(A 4)$ Conditions $\left(C_{3}\right)$ and $f_{\infty} \in\left(\left(\frac{\rho^{*}}{\sigma}\right)^{p-1}, \infty\right)\left(\right.$ or $\left.f_{0} \in\left(\left(\frac{\rho^{*}}{\sigma}\right)^{p-1}, \infty\right)\right)$ hold; or

(A5) Conditions $\left(C_{4}\right)$ and $f^{0} \in\left[0, \rho_{*}^{p-1}\right)\left(\right.$ or $\left.f^{\infty} \in\left[0, \rho_{*}^{p-1}\right)\right)$ hold.

\section{Examples}

In this section, we present some examples to check our results. Let

$$
\alpha=\frac{5}{2}, \quad \beta=\frac{3}{2}, \quad q=2, \quad p=2, \quad \xi=\frac{1}{2}, \quad \lambda=\frac{\sqrt{2}}{2},
$$

then there are

$$
H(x, z)= \begin{cases}\frac{1}{\sqrt{\pi}}\left\{4[x(1-z)]^{\frac{1}{2}}-2 \sqrt{2}\left[x\left(\frac{1}{2}-z\right)\right]^{\frac{1}{2}}-2(x-z)^{\frac{1}{2}}\right\}, & 0 \leq z \leq x \leq 1, z \leq \frac{1}{2} \\ \frac{1}{\sqrt{\pi}}\left\{4[x(1-z)]^{\frac{1}{2}}-2(x-z)^{\frac{1}{2}}\right\}, & 0<\frac{1}{2} \leq z \leq x \leq 1 ; \\ \frac{1}{\sqrt{\pi}}\left\{4[x(1-z)]^{\frac{1}{2}}-2 \sqrt{2}\left[x\left(\frac{1}{2}-z\right)\right]^{\frac{1}{2}}\right\}, & 0 \leq x \leq z \leq \frac{1}{2}<1 ; \\ \frac{4}{\sqrt{\pi}}[x(1-z)]^{\frac{1}{2}}, & 0 \leq x \leq z \leq 1, \frac{1}{2} \leq z,\end{cases}
$$

and

$$
\begin{aligned}
& M=\lambda^{p-1} \xi^{\beta-1}=\frac{1}{2}, \quad Q(x)=x(1-x)^{\alpha-1}=x(1-x)^{\frac{3}{2}}, \\
& \rho^{*}=22.5, \quad \rho_{*}=16, \quad \sigma=\frac{1}{3} .
\end{aligned}
$$

Example 4.1 Consider the boundary value problem

$$
\begin{aligned}
& D_{0+}^{\beta}\left(\varphi_{p}\left(D_{0+}^{\alpha} y(x)\right)\right)=f(x, y(x)), \quad 0<x<1, \\
& y(0)=y^{\prime}(0)=y(1)=D_{0+}^{\alpha} y(0)=0, \quad D_{0+}^{\alpha} y(1)=\lambda D_{0+}^{\alpha} y(\xi),
\end{aligned}
$$

where

$$
f(x, y)=\frac{y^{2}}{2} \cos x+70 \sin y
$$

Direct computations show that

$$
\begin{aligned}
& f_{0}=\liminf _{u \rightarrow 0+} \min _{x \in[1 / 4,3 / 4]} \frac{f(x, y)}{y}=\liminf _{y \rightarrow 0+}\left(\frac{y \cos \frac{3}{4}}{2}+70 \frac{\sin y}{y}\right)=70>68=\left(\frac{\rho^{*}}{\sigma}\right)^{p-1}, \\
& f_{\infty}=\liminf _{y \rightarrow+\infty} \min _{x \in[1 / 4,3 / 4]} \frac{f(x, y)}{y}=\liminf _{y \rightarrow+\infty}\left(\frac{y \cos \frac{3}{4}}{2}+70 \frac{\sin y}{y}\right)=\infty>68=\left(\frac{\rho^{*}}{\sigma}\right)^{p-1},
\end{aligned}
$$


so condition $\left(C_{1}\right)$ holds. Choose $\lambda_{1}=6, d=15 \in\left(0, \rho_{*}\right)$, one has

$$
f(x, y) \leq 88<90=d \lambda_{1}, \quad \text { when } 0 \leq x \leq 1,0 \leq y \leq 6
$$

so condition $\left(C_{3}\right)$ holds. By the use of Theorem 3.1, problem (1.1) has at least two solutions $y_{1}$ and $y_{2}$ satisfying $0<\left\|y_{1}\right\|<6<\left\|y_{2}\right\|$.

Example 4.2 Consider the boundary value problem (4.1), (4.2), where

$$
f(x, y)=\frac{500 y \sin y}{y+1}+15 x y \text {. }
$$

Direct computations show that

$$
\begin{aligned}
& f^{0}=\underset{y \rightarrow 0+}{\limsup } \max _{x \in[0,1]} \frac{f(x, y)}{y}=\limsup _{y \rightarrow 0+}\left(\frac{500 \sin y}{y+1}+15\right)=15<16=\rho_{*}^{p-1}, \\
& f^{\infty}=\limsup _{y \rightarrow+\infty} \max _{x \in[0,1]} \frac{f(x, y)}{y}=\limsup _{y \rightarrow+\infty}\left(\frac{500 \sin y}{y+1}+15\right)=15<16=\rho_{*}^{p-1},
\end{aligned}
$$

so condition $\left(C_{2}\right)$ holds. Choose $\lambda_{2}=\frac{1}{2}, D=23 \in\left(\rho^{*}, \infty\right)$, one has

$$
f(x, y) \geq 12.4>D \lambda_{2}=\frac{23}{2}, \quad \text { for } \frac{1}{4} \leq x \leq \frac{3}{4}, \frac{1}{6}=\sigma \lambda_{2} \leq y \leq \lambda_{2}=\frac{1}{2}
$$

so condition $\left(C_{4}\right)$ holds. By the use of Theorem 3.2, problem (1.1) has at least two solutions $y_{1}$ and $y_{2}$ satisfying $0<\left\|y_{1}\right\|<\frac{1}{2}<\left\|y_{2}\right\|$.

Example 4.3 Consider the boundary value problem (4.1), (4.2), where

$$
f(x, y)=5+x y^{2}
$$

Let $\lambda_{1}=2, d=5 \in\left(0, \rho_{*}\right)$. Direct computations show that

$$
f(x, y) \leq 9<d \lambda_{1}=10, \quad \text { for } 0 \leq x \leq 1,0 \leq y \leq 2,
$$

and

$$
f_{\infty}=\liminf _{y \rightarrow+\infty} \min _{x \in[1 / 4,3 / 4]} \frac{f(x, y)}{y}=\liminf _{y \rightarrow+\infty}\left(\frac{5}{y}+\frac{y}{4}\right)=\infty>68=\left(\frac{\rho^{*}}{\sigma}\right)^{p-1} .
$$

So condition (A4) was satisfied. By the use of Corollary 3.1, problem (1.1) has at least one positive solution.

Acknowledgements

Not applicable.

Funding

This work is supported by the Scientific Research Foundation of Hunan Provincial Education Department (16A198), the Hunan Provincial Natural Science Foundation of China (2015JJ6101), the Construct Program of the Key Discipline in Hunan Province, and the NSFC (11571207). 
Availability of data and materials

Data sharing not applicable to this article as no data sets were generated or analysed during the current study.

\section{Competing interests}

The authors declare that they have no competing interests.

\section{Authors' contributions}

All authors contributed equally to the writing of this paper. All authors read and approved the final manuscript.

\section{Authors' information}

Yuanshen Tian, professor, his main research field is fractional differential equation boundary value problem. Yongfang Wei and Sujing Sun, doctoral candidates, their research fields are w.r.t. the application of nonlinear functional analysis on differential equations.

\section{Author details}

${ }^{1}$ College of Mathematics and Finance, Xiangnan University, Chenzhou, P.R. China. ${ }^{2}$ College of Mathematics and System Science, Shandong University of Science and Technology, Qingdao, P.R. China.

\section{Publisher's Note}

Springer Nature remains neutral with regard to jurisdictional claims in published maps and institutional affiliations.

\section{Received: 2 May 2018 Accepted: 6 August 2018 Published online: 16 August 2018}

\section{References}

1. Bai, Z:: Solvability for a class of fractional m-point boundary value problem at resonance. Comput. Math. Appl. 62, 1292-1302 (2011)

2. Bai, Z., Dong, X., Yin, C.: Existence results for impulsive nonlinear fractional differential equation with mixed boundary conditions. Bound. Value Probl. 2016, Article ID 63 (2016). https://doi.org/10.1186/s13661-016-0573-z

3. Bai, Z., Qiu, T.: Existence of positive solution for singular fractional differential equation. Appl. Math. Comput. 215, 2761-2767 (2009)

4. Bai, Z., Zhang, S., Sun, S., Yin, C.: Monotone iterative method for fractional differential equations. Electronic Journal of Differential Equations 2016, Article ID 6 (2016)

5. Bai, Z., Zhang, Y.: Solvability of fractional three-point boundary value problems with nonlinear growth. Appl. Math. Comput. 218, 1719-1725 (2011)

6. Cabada, A., Wang, G.: Positive solutions of nonlinear fractional differential equations with integral boundary value conditions. Abstr. Appl. Anal. 2012, 403 (2012)

7. Cui, Y.: Uniqueness of solution for boundary value problems for fractional differential equations. Appl. Math. Lett. 51 48-54 (2016)

8. Cui, Y., Sun, J.: A generalization of Mahadevan's version of the Krein-Rutman theorem and applications to $p$-Laplacian boundary value problems. Abstr. Appl. Anal. 2012, Article ID 305279 (2012). https://doi.org/10.1155/2012/305279

9. Dong, H., Guo, B., Yin, B.: Generalized fractional supertrace identity for Hamiltonian structure of NLS-MKdV hierarchy with self-consistent sources. Anal. Math. Phys. 6, 199-209 (2016)

10. Dong, X., Bai, Z., Zhang, S.: Positive solutions to boundary value problems of $p$-Laplacian with fractional derivative Bound. Value Probl. 2017, Article ID 5 (2017). https://doi.org/10.1186/s13661-016-0735-z

11. Fu, C., Lu, C., Yang, H.: Time-space fractional $(2+1)$ dimensional nonlinear Schrodinger equation for envelope gravity waves in baroclinic atmosphere and conservation laws as well as exact solutions. Adv. Differ. Equ. 2018, Article ID 56 (2018)

12. Hao, X.: Positive solution for singular fractional differential equations involving derivatives. Adv. Differ. Equ. 2016, Article ID 139 (2016). https://doi.org/10.1186/s13662-016-0865-8

13. Hao, X., Wang, H.: Positive solutions of semipositone singular fractional differential systems with a parameter and integral boundary conditions. Open Math. 16, 581-596 (2018)

14. Hao, X., Wang, H., Liu, L., Cui, Y.: Positive solutions for a system of nonlinear fractional nonlocal boundary value problems with parameters and p-Laplacian operator. Bound. Value Probl. 2017, Article ID 182 (2017)

15. Hao, X., Zuo, M., Liu, L.: Multiple positive solutions for a system of impulsive integral boundary value problems with sign-changing nonlinearities. Appl. Math. Lett. 82, 24-31 (2018)

16. Ji, D., Bai, Z., Ge, W.: The existence of countably many positive solutions for singular multipoint boundary value problems. Nonlinear Anal., Theory Methods Appl. 72, 955-964 (2010)

17. Kilbas, A., Srivastava, H., Trujillo, J.: Theory and Applications of Fractional Differential Equations. Elsevier, Amsterdam (2006)

18. Krasnosel'skii, M.: Positive Solutions of Operator Equations. Noordhoff, Groningen (1964)

19. Leibenson, L.: Turbulent movement of gas in a porous medium. Izv. Akad. Nauk SSSR, Ser. Geogr. Geofiz. 9, 3-6 (1945) (in Russian)

20. Li, H., Sun, J.: Positive solutions of superlinear semipositone nonlinear boundary value problems. Comput. Math. Appl. 61, 2806-2815 (2011)

21. Liu, X., Jia, M., Ge, W.: Multiple solutions of a p-Laplacian model involving a fractional derivative. Adv. Differ. Equ. 2013, $1(2013)$

22. Liu, X., Jia, M., Ge, W.: The method of lower and upper solutions for mixed fractional four-point boundary value problem with $p$-Laplacian operator. Appl. Math. Lett. 65, 56-62 (2017)

23. Lu, C., Fu, C., Yang, H.: Time-fractional generalized Boussinesq equation for Rossby solitary waves with dissipation effect in stratified fluid and conservation laws as well as exact solutions. Appl. Math. Comput. 327, 104-116 (2018) 
24. LV, W., Wang, F.: Adaptive tracking control for a class of uncertain nonlinear systems with infinite number of actuator failures using neural networks. Adv. Differ. Equ. 2017, 374 (2017)

25. Song, Q., Dong, X., Bai, Z., Chen, B.: Existence for fractional Dirichlet boundary value problem under barrier strip conditions. J. Nonlinear Sci. Appl. 10, 3592-3598 (2017)

26. Su, H., Wei, Z., Wang, B.: The existence of positive solutions for a nonlinear four-point singular boundary value problem with a $p$-Laplacian operator. Nonlinear Anal. 66, 2204-2217 (2007)

27. Tang, X.: Existence of solutions of four-point boundary value problems for fractional differential equations at resonance. J. Appl. Math. Comput. 51, 145-160 (2016)

28. Tian, Y.: Positive solutions to $m$-point boundary value problem of fractional differential equation. Acta Math. Appl. Sin. $29,661-672(2013)$

29. Tian, Y., Sun, S., Bai, Z.: Positive solutions of fractional differential equations with $p$-Laplacian. Journal of Function Spaces 2017, Article ID 3187492 (2017). https://doi.org/10.1155/2017/3187492

30. Wang, G., Agarwal, R., Cabada, A.: Existence results and monotone iterative technique for systems of nonlinear fractional differential equations. Appl. Math. Lett. 25(6), 1019-1024 (2012)

31. Wang, J., Zhou, Y., Wei, W., Xu, H.: Nonlocal problems for fractional integrodifferential equations via fractional operators and optimal controls. Comput. Math. Appl. 62, 1427-1441 (2011)

32. Wang, Y., Hou, C.: Existence of multiple positive solutions for one dimensional p-Laplacian. J. Math. Anal. Appl. 315, 144-153 (2006)

33. Wang, Z.: A numerical method for delayed fractional-order differential equations. J. Appl. Math. 2013, Article ID 256071 (2013). https://doi.org/10.1155/2013/256071

34. Wang, Z., Huang, X., Shi, G.: Analysis of nonlinear dynamics and chaos in a fractional order financial system with time delay. Comput. Math. Appl. 62, 1531-1539 (2011)

35. Wei, Y., Song, Q., Bai, Z.: Existence and iterative method for some fourth order nonlinear boundary value problems. Appl. Math. Lett. 87, 101-107 (2019)

36. Yan, F., Zuo, M., Hao, X.: Positive solution for a fractional singular boundary value problem with $p$-Laplacian operator. Bound. Value Probl. 2018, Article ID 51 (2018). https://doi.org/10.1186/s13661-018-0972-4

37. Yang, Y., LV, L., Wang, J.: Existence results for boundary value problems of high order differential equations involving Caputo derivative. J. Appl. Math. Comput. 38, 565-583 (2012)

38. Zhang, W., Bai, Z., Sun, S.: Extremal solutions for some periodic fractional differential equations. Adv. Differ. Equ. 2016, Article ID 179 (2016). https://doi.org/10.1186/s13662-016-0869-4

39. Zou, Y., Cui, Y.: Existence results for a functional boundary value problem of fractional differential equations. Adv. Differ. Equ. 2013, Article ID 233 (2013). https://doi.org/10.1186/1687-1847-2013-233

40. Zou, Y., Liu, L., Cui, Y.: The existence of solutions for four-point coupled boundary value problems of fractional differential equations at resonance. Abstr. Appl. Anal. 2014, Article ID 314083 (2014). https://doi.org/10.1155/2014/314083

41. Zuo, M., Hao, X., Liu, L., Cui, Y.: Existence results for impulsive fractional integro-differential equation of mixed type with constant coefficient and antiperiodic boundary conditions. Bound. Value Probl. 2017, Article ID 61 (2017). https://doi.org/10.1186/s13661-017-0892-8

\section{Submit your manuscript to a SpringerOpen ${ }^{\circ}$ journal and benefit from:}

- Convenient online submission

- Rigorous peer review

- Open access: articles freely available online

- High visibility within the field

- Retaining the copyright to your article

Submit your next manuscript at $\gg$ springeropen.com 